\title{
Kinematic properties of superbubbles in the Antennae, M83 and Arp 270
}

\author{
A. Camps-Fariña ${ }^{1,2}$, J. Beckman ${ }^{1,2,3}$, J. Zaragoza-Cardiel ${ }^{1,2}$, J. Font ${ }^{1,2}$, \\ K. Fathi ${ }^{4}$, P. Fabian Velázquez ${ }^{5}$ and A. Rodríguez-González ${ }^{5}$ \\ ${ }^{1}$ Instituto de Astrofísica de Canarias, La Laguna, Spain \\ ${ }^{2}$ Departamento de Astrofísica, Universidad de la Laguna, Spain \\ ${ }^{3}$ Consejo Superior de Investigaciones Científicas, Spain \\ ${ }^{4}$ Department of Astronomy, Stockholm University, Stockholm, Sweden \\ ${ }^{5}$ Instituto de Ciencias Nucleares, Universidad Nacional de México, D.F, Mexico
}

\begin{abstract}
Superbubbles and large scale expansion in galaxies are important indicators of activity in galaxies: they are formed in starbursts and around active nuclei. Superbubbles can be used to give information about the star-forming region which produced them. We present in-depth results of our study of kinematically detected superbubbles using a method based on Fabry-Perot spectroscopy, which allows us to map regions of expansion across the entire disk of a galaxy. Three objects have been selected for this poster based on the interest of the results they show: two interacting galaxies, the Antennae and Arp270, at different stages of galaxy interaction, and the more isolated galaxy M83. We present the kinematic expansion maps, as well as a census of detected superbubbles and a dynamical study of their properties.
\end{abstract}

Keywords. galaxies: superbubbles, galaxies: kinematics, galaxies: evolution

We use the method described in the poster Fabry-Perot spectroscopy: a powerful method for detecting superbubbles in galaxy discs to derive expansion maps of three galaxies and detect on these the presence of superbubbles. We find 10 superbubbles in the Antennae galaxies (Arp 244), 8 in M83 and 3 in the central zones of the interacting pair Arp 270. We measure their properties: radius, expansion velocity, $\mathrm{H} \alpha$ luminosity, density, mass, kinetic energy, and an approximate age. To assess the validity of our results we use hydrodynamic simulations to reproduce a well-characterised superbubble from its measured properties. The simulation successfully reproduces the bubble, in Fig. 1 we present the expansion map around the bubble and the equivalent measurement from the simulated bubble.
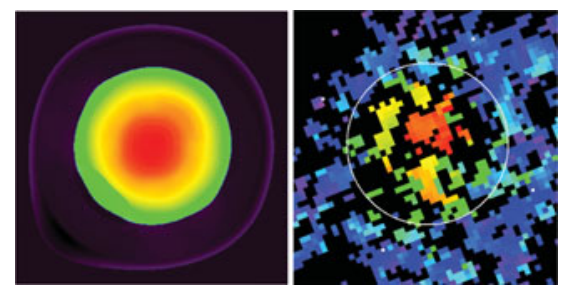

Figure 1. Comparison between a detected superbubble in the Antennae (right) and the equivalent projection of a hydrodynamical simulation done using its measured parameters. Colors represent observed line of sight expansion velocity from maximum in red through lower green-blue.

\section{References}

Raga, A. C., Navarro-González, R., \& Villagrán-Muniz, M., 2000, RMxAA, 36, 67 